\title{
BMJ Open Effects of home-based cardiac exercise rehabilitation with remote electrocardiogram monitoring in patients with chronic heart failure: a study protocol for a randomised controlled trial
}

Jiahui Li, ${ }^{1}$ Peng Yang, ${ }^{2}$ Dongliang Fu, ${ }^{2}$ Xiaojun Ye, ${ }^{1}$ Lifang Zhang, ${ }^{1}$ Gang Chen, ${ }^{3}$ Yiyun Yang, ${ }^{1} \mathrm{He}$ Luo, ${ }^{1} \mathrm{Li}$ Chen, ${ }^{4}$ Mingjing Shao, ${ }^{2}$ Chunyan Li, ${ }^{2}$ Yi Liu, ${ }^{2}$ Ying Zhou, ${ }^{1}$ Hong Jiang, ${ }^{2}$ Xianlun $\mathrm{Li}^{1,2}$

To cite: Li J, Yang P, Fu D, et al. Effects of home-based cardiac exercise rehabilitation with remote electrocardiogram monitoring in patients with chronic heart failure: a study protocol for a randomised controlled trial. BMJ Open 2019:9:e023923. doi:10.1136/ bmjopen-2018-023923

- Prepublication history for this paper is available online. To view these files, please visit the journal online (http://dx.doi org/10.1136/bmjopen-2018023923).

Received 9 May 2018 Revised 4 December 2018 Accepted 7 December 2018

Check for updates

(C) Author(s) (or their employer(s)) 2019. Re-use permitted under CC BY-NC. No commercial re-use. See rights and permissions. Published by BMJ.

For numbered affiliations see end of article.

\section{Correspondence to}

Dr Hong Jiang;

drjh68@163.com and Dr Xianlun $\mathrm{Li}$;

leexianlun@163.com

\begin{abstract}
Introduction Patients with chronic heart failure (CHF) can benefit from exercise rehabilitation (ER) with significant improvements in exercise capacity, quality of life and reduction in hospitalisations. Despite its reported benefits, only a small number of patients with CHF attend ER due to poor adherence, and improper exercise may even lead to adverse events. Remote ECG monitoring system (REMS) has the potential to overcome these obstacles. We hypothesise that home-based cardiac ER using REMS in CHF patients is effective compared with conventional ER without monitoring.

Methods and analysis This study is a prospective, randomised, parallel controlled clinical trial designed to evaluate the effectiveness of home-based phase-II ER with REMS in the treatment of CHF with a target enrolment of 120 patients (left ventricular ejection fraction $<50 \%$, New York Heart Association (NYHA) classes I to III). Patients are randomised to either REMS rehabilitation group or conventional rehabilitation group in a 1:1 ratio. All patients

Strengths and limitations of this study

- The wearable monitor used in this study is portable, which can evaluate patients' exercise intensity based on their heart rate and then remind them to exercise appropriately.

- The remote ECG monitoring system used here can detect arrhythmia and provide an early warning to both the exercising patients and their doctors to further reduce risks.

- Our cardiac exercise rehabilitation (ER) programme is home based and convenient for patients with chronic heart failure (CHF) compared with facility-based ER, which may provide new insights into cardiac telerehabilitation in patients with CHF.

- The main limitation of this single-centre study is the small sample size.

- A long-term follow-up may also be needed, and extending the exercise for 6 months may give us better data on adherence.
\end{abstract} start an exercise training in a supervised setting and then transition to a home-based regimen. The supervised training phase consists of 12 supervised training sessions, three sessions per week for 4 weeks. During the home exercise phase, patients exercise five times per week for 8 weeks. In the REMS group, patients wear monitors during exercise to ensure that exercise intensity is within the set ranges. REMS will also detect risky arrhythmia and alert the patients and their doctors on time. The training intensity is not monitored in the conventional rehabilitation group. The primary outcome is exercise capacity improvement measured by peak oxygen uptake $\left(\mathrm{VO}_{2}\right.$ peak $)$ (baseline vs $3 \mathrm{~m}$ ). Secondary outcomes include 6-min walk test, NYHA classes, echocardiographic parameters, cardiac biomarkers, major adverse cardiovascular events, quality of life, psychological well-being and patients' adherence to the rehabilitation programme.

Ethics and dissemination This study was approved by Ethics Committee of China-Japan Friendship Hospital for Clinical Research (No. 2018-55 K39). The results of this study will be disseminated via peer-reviewed publications and presentations at conferences.

Trial registration number ChiCTR-RNR-17012446; Preresults.

\section{INTRODUCTION}

Heart failure (HF) is a complex clinical syndrome caused by abnormal cardiac structure or function which results in impaired ventricular filling or ejection ability. It is a terminal stage of various cardiovascular diseases, which has become the global public health problem of great concern. The prevalence of $\mathrm{HF}$ in the entire population is approximately $1.5 \%-2 \%$, and $6 \%-10 \%$ above 65 years of age. ${ }^{1}$ Nowadays, due to 
modern therapies, more and more people are spared from acute cardiovascular attacks, and the concomitant burden of chronic HF (CHF) is also increasing globally. According to the 2017 China Cardiovascular report, ${ }^{2}$ there are currently $8-10$ million HF patients in China, and the prevalence rate increases significantly with age. The 5-year survival rate of HF patients with clinical symptoms is $50 \%$, similar to that of malignant tumours. ${ }^{1}$ For HF patients, repeated visits and hospitalisations place heavy financial burdens on individuals, families and society.

Many HF patients on optimal cardiovascular medical therapy still suffer from dyspnoea and exercise intolerance. In addition to medical treatment and device therapy, successful cardiac rehabilitation (CR) is a valuable intervention for improving aerobic fitness and overall health status in patients with HF. HF patients can benefit from proper exercise with significant improvements in exercise capacity, quality of life and reduction in hospitalisations. ${ }^{3-6}$ Exercise training is a core component of primary and secondary prevention for HF, which has been recommended by relevant international professional associations. ${ }^{3-6}$

Despite its reported benefits, a limited number of $\mathrm{HF}$ patients regularly participate in exercise rehabilitation (ER), primarily because of poor patients' adherence. In addition, inappropriate exercise that is not monitored may even cause adverse cardiovascular events such as myocardial infarction, arrhythmias and sudden death. HF-A Controlled Trial Investigating Outcomes of exercise training $^{7}$ is the largest multicentre clinical study of ER in HF, which randomly assigned 2331 patients to either exercise training or usual care. Only $42 \%$ of the subjects in their cohort completed all three scheduled follow-up exercise tests and $33 \%$ and $25 \%$ completed two and one of their scheduled follow-up exercise tests, respectively. Given the observed effect between higher adherence and improved outcomes, it is more important to provide cardiac ER programmes which can achieve increased adherence to the exercise intervention. ${ }^{8}$

Cardiac telerehabilitation using monitoring devices and remote communication with patients is now increasingly used for long-term management of cardiovascular diseases outside the hospital environment. Providing objective feedback data and allowing patients to track their own progress can improve patients' self-management skills and thereby improve their adherence. It is convenient, can reduce anxiety, and improve the quality of life and prognosis of patients with low medical costs compared with conventional ER without monitoring. ${ }^{9-13}$

This study is a prospective, randomised, parallel controlled clinical trial to evaluate the effectiveness and safety of home-based phase-II ER under the guidance of remote ECG monitoring system (REMS) in the management of CHF compared with conventional ER without monitoring. This paper describes the design and rationale of the Home-based Exercise Rehabilitation with Electrocardiogram (HERE)-CHF trial.

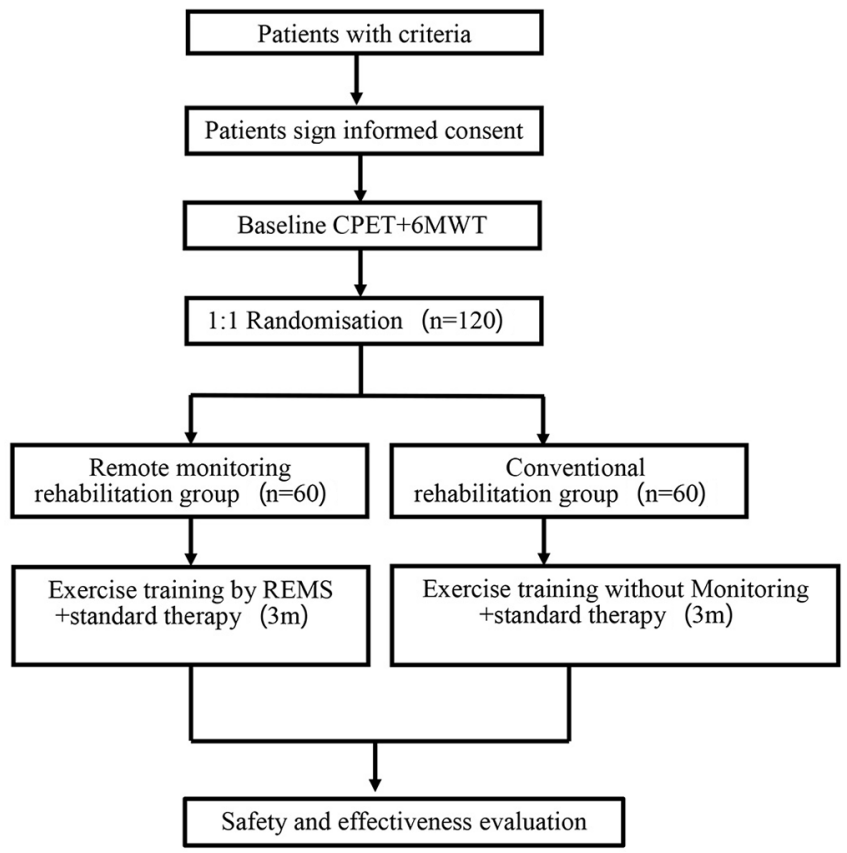

Figure 1 Study flow chart. 6MWT, 6 min walk test; CPET, cardiopulmonary exercise testing; REMS, remote ECG monitoring system.

\section{METHODS/DESIGN \\ Design}

A flow chart of the study design is shown in figure 1 . The protocol is constructed and presented in accordance with the Standard Protocol Items: Recommendations for Interventional Trials. ${ }^{14}$ The study protocol (V1.1, 20180208) and informed consent documents (V1.1, 20180208) have been reviewed and approved by the Ethics Committee of China-Japan Friendship Hospital for Clinical Research. If there is any amendment to the protocol, approval must be sought again from the Ethics Committee. The study strategy has been registered on the website of Chinese Clinical Trial Registry (ChiCTR-RNR-17012446, registered on 22 August 2017), and the trial will be conducted in accordance with the principles of the Declaration of Helsinki and Good Clinical Practice guidelines.

\section{Eligibility and recruitment}

Patient selection criteria are listed in box 1. Patients must have a systolic cardiac dysfunction documented from a baseline echocardiogram (left ventricular ejection fraction $<50 \%$ ) and are in stable conditions (New York Heart Association (NYHA) classes I to III)within 6 weeks prior to randomisation. According to the American College of Cardiology /American Heart Association (AHA) HF guidelines, stable optimal drug therapies including $\beta$-blockers, diuretics, angiotensin-converting enzyme inhibitors or angiotensin II receptor blockers and aldosterone receptor antagonists for 6 weeks before enrolment are strongly recommended. If patients are not treated with optimal medical therapy, then trial personnel must document the underlying reason (eg, drug intolerance). 
Box 1 HERE-CHF trial inclusion and exclusion criteria

\section{Inclusion criteria}

1. Aged $18-75$ years old.

2. NYHA I-III; LVEF $<50 \%$ in 6 weeks before randomisation.

3. In stable condition after heart failure standard drug therapy (do not need intravenous diuretics, vasoconstrictors, vasodilators or left ventricular assist device), no fluid retention, constant weight.

4. Able to perform exercise rehabilitation.

5. Clearly understand the content and purpose of the study and volunteer to participate in the study and sign the informed consent form.

\section{Exclusion criteria}

1. Exercise rehabilitation cannot be carried out due to physical disability and contraindication.

2. With a contraindication to cardiopulmonary exercise test.

3. The following was happened in 3 months before admission: acute coronary syndrome, stroke, transient ischaemic attack; cardiac, carotid artery or other major vascular surgery; percutaneous coronary intervention or carotid artery angioplasty; sustained ventricular tachycardia or fibrillation.

4. Coronary revascularisation or heart transplantation is planned.

5. Persistent atrial arrhythmias; ventricular arrhythmias were not effectively controlled (using an antiarrhythmic drug or an implantable defibrillator).

6. Uncorrected primary obstructive or severe regurgitant heart valve disease, restrictive or hypertrophic cardiomyopathy.

7. II or III degree heart block or sick sinus syndrome without permanent pacemaker implantation; need implantable device therapy for heart failure.

8. Obstructive or bronchospasm lung diseases (such as asthma, bronchitis, etc) needing oral or inhaled bronchodilator or glucocorticoids therapy.

9. Pregnant or lactating women and those planning to conceive during the trial.

10. Cancer or other systemic diseases with an expected survival of less than 12 months.

11. Other clinical trial drugs were taken or in other medical device trial within 30 days before admission.

12. Unable to participate in this study after the clinical evaluation by investigators.

NYHA, New York Heart Association; LVEF, left ventricular ejection fraction.NYHA, New York Heart Association; LVEF, left ventricular ejection fraction.

Patients enrolment began in April 2018. Patients were recruited consecutively from the outpatient service of Cardiology Department and Integrative Cardiology Department in China-Japan Friendship Hospital by attending physicians responsible for recruitment, who had obtained written consent from patients who were willing to participate in the trial. Screening will continue until the target population is achieved. A recruitment advertisement has also been utilised in our hospital. No financial incentives were provided to the attending physicians or patients for enrolment.

\section{Sample size}

The target number of participants in the trial is 120 subjects. A total of $500 \mathrm{HF}$ patients (NYHA grades I-III) are treated in our hospital each year and can be used as a screening sample. Based on the lower screening rate $(50 \%)$ and the designed entry criteria, the sample size that can be completed is estimated to be 100 patients. With reference to previous foreign literature, the compliance of ER within 3 months is $70 \%-80 \%$. The study will enrol at least 120 subjects, 60 subjects in each group.

\section{Exercise testing}

The pre-randomisation cardiopulmonary exercise testing (CPET) is used to determine whether patients can exercise safely in accordance with the guidelines of the American Association of Cardiovascular and Pulmonary Rehabilitation, including checking for abnormal blood pressure responses, early ischaemic changes and significant arrhythmias. Exercise testing is repeated 3 months after randomisation for all patients. The primary method used for exercise testing is cycling, consistent with AHA guidelines and with other trials that have assessed exercise capacity in patients with HF.

\section{Randomisation and binding}

The clinical research data management platform of China-Japan Friendship Hospital was commissioned to generate a random sequence of 120 numbers using SAS 9.4 software. The random sequence is placed into a sealed envelope by a staff member who is not involved in the study to avoid selection bias. After providing informed consent and undergoing baseline testing (echocardiogram and CPET), patients are randomly assigned to the REMS rehabilitation group or the conventional rehabilitation group in a 1:1 ratio based on the patient's admission time. Allocation concealment is ensured and the randomisation code will not be released until the patient has been recruited into the trial, which takes place after all baseline measurements have been completed. Researchers involved in participants' assessments are blinded to treatment allocation.

\section{Trial structure}

The trial structure of the study is described in table 1 .

\section{Remote ECG monitoring system}

Inticare-MC-06 wearable ECG monitor (Elephant Medical Electronic Technology Co., Ltd, Jining, China), approved by Food and Drug Administration in Shandong province of China (Certificate No. Shandong Medical Device Registration Approval 20172210878), is a medical-grade portable cardiac monitor and looks like a smaller 'Band-Aid' that can collect 48 hours of single-lead ECG data. It can provide cardiac care service through a 'Hardware +Software+ Cloud+Doctor' program, and support arrhythmia automatic trigger and One-Tap SOS. Inticare-MC-06 heart health monitor solution consists of five parts: a wearable ECG monitor, mobile terminals (such as smart phones), Cardiac Healthcare Cloud Service platform of Elephant Medical (Tianjin AI-Life Medical Technology, China), cardiologists and monitoring reports, supported by Tianjin Institute of Internet of Things Technology. The wearable ECG monitor is pasted on the chest 
Table 1 Trial structure of the study

\begin{tabular}{|c|c|c|c|c|c|c|}
\hline \multirow[b]{2}{*}{ Time } & \multirow[b]{2}{*}{$\begin{array}{l}\text { Screening } \\
(-14 \sim-1 d)\end{array}$} & \multicolumn{3}{|c|}{ Exercise rehabilitation in clinic } & \multicolumn{2}{|c|}{ Exercise rehabilitation at home } \\
\hline & & $\begin{array}{l}\text { T0 } \\
\text { Visit 1 } \\
\text { Baseline }\end{array}$ & $\begin{array}{l}\text { T1 } \\
\text { Visit } 2 \\
\text { Second weekend }\end{array}$ & $\begin{array}{l}\text { T2 } \\
\text { Visit } 3 \\
\text { Fourth weekend }\end{array}$ & $\begin{array}{l}\text { T3 } \\
\text { Visit } 4 \\
\text { Eighth weekend }\end{array}$ & $\begin{array}{l}\text { T4 } \\
\text { Visit } 5 \\
\text { 12th weekend }\end{array}$ \\
\hline \multicolumn{7}{|l|}{ Assessment } \\
\hline Medical history & $\sqrt{ }$ & & & & & \\
\hline Inclusion/exclusion form & $\sqrt{ }$ & & & & & \\
\hline Consent form & $\sqrt{ }$ & & & & & \\
\hline Comorbidity & $\sqrt{ }$ & $\sqrt{ }$ & $\sqrt{ }$ & $\sqrt{ }$ & $\sqrt{ }$ & $\sqrt{ }$ \\
\hline Concomitant medication & $\sqrt{ }$ & $\sqrt{ }$ & $\sqrt{ }$ & $\sqrt{ }$ & $\sqrt{ }$ & $\sqrt{ }$ \\
\hline Physical examination & $\sqrt{ }$ & $\sqrt{ }$ & $\sqrt{ }$ & $\sqrt{ }$ & $\sqrt{ }$ & $\sqrt{ }$ \\
\hline Troponin T/I & & $\sqrt{ }$ & & & & $\sqrt{ }$ \\
\hline BNP/NT-proBNP & & $\sqrt{ }$ & & & & $\sqrt{ }$ \\
\hline ECG & $\sqrt{ }$ & $\sqrt{ }$ & & $\sqrt{ }$ & $\sqrt{ }$ & $\sqrt{ }$ \\
\hline Holter & $\sqrt{ }$ & & & & & $\sqrt{ }$ \\
\hline NYHA classification & $\sqrt{ }$ & $\sqrt{ }$ & & $\sqrt{ }$ & $\sqrt{ }$ & $\sqrt{ }$ \\
\hline Echocardiography & $\sqrt{ }$ & $\sqrt{ }$ & & & & $\sqrt{ }$ \\
\hline CPET & $\sqrt{ }$ & & & & & $\sqrt{ }$ \\
\hline 6MWT & & $\sqrt{ }$ & & & & $\sqrt{ }$ \\
\hline Heart failure Symptom Scale & & $\sqrt{ }$ & & & & $\sqrt{ }$ \\
\hline MLHFQ & & $\sqrt{ }$ & & & & $\sqrt{ }$ \\
\hline SF-36 & & $\sqrt{ }$ & & & & $\sqrt{ }$ \\
\hline BDI-II & & $\sqrt{ }$ & & & & $\sqrt{ }$ \\
\hline GSE & & $\sqrt{ }$ & & & & $\sqrt{ }$ \\
\hline Compliance & & & & $\sqrt{ }$ & $\sqrt{ }$ & $\sqrt{ }$ \\
\hline Adverse event & & & $\sqrt{ }$ & $\sqrt{ }$ & $\sqrt{ }$ & $\sqrt{ }$ \\
\hline Monitor and APP training & & $\sqrt{ }$ & & & & \\
\hline
\end{tabular}

The time window for each visit is $\pm 3 \mathrm{~d}$.

6MWT, 6 min walk test; BDI-II, Beck Depression Inventory; BNP/NT, B-type natriuretic peptide/N-terminal; GSE, General Self-Efficacy Scale; MLHFQ, Minnesota Living with Heart Failure Questionnaire; NYHA, New York Heart Association; SF-36, 36-Item Short Form Health Survey.

through one-piece electrodes and transfers the ECG data to the smart phone application of cloud service platform (Elephant Heart Health) via bluetooth smart technology. If an ECG such as arrhythmia occurs during exercise, the system will alert the patient to stop or suspend exercise, or adjust the intensity of exercise, and upload it in real time. The data will be transformed to the data centre, and the specialist will analyse that report. The unique algorithm analyses the data in real time and detects suspicious arrhythmias, then sends out alerts and transfers data to cloud platform for doctors to check and confirm the disease. In addition, when patients have chest discomfort, palpitation and dizziness, they can mark the ECG data through One-Tap Marking button and send the data for doctors to check and give corresponding diagnosis. At the end of the monitoring, a report will be provided.

\section{Interventions}

All the patients initiate an exercise training programme in accordance with the principles of exercise prescription recommended by the American College of Sports Medicine and the AHA. The sequence of the exercise phase can be warm-up, main exercise and cool-down. For the warm-up and cool-down, walking or light stretching is recommended. The main exercise is walking. Exercise training initially begins in a supervised setting and then transitions to a home-based regimen (tables 2 and 3 ). The supervised training phase consists of 12 supervised training sessions, with a goal of three sessions per week. It takes up to 1 month for patients to complete the 12 sessions before transition to the home exercise phase. Patients are required to exercise five times per week during the home exercise phase for a total of 8 weeks. Patients begin exercising at a low intensity and then increase to a moderate intensity when they are able to do so. The trial protocol allows patients to walk independently.

In the REMS group, patients wear Inticare-MC-06 ECG monitors while exercising at home to ensure that exercise intensity is within their set ranges and are instructed how 
Table 2 Exercise training programme in remote ECG monitoring system rehabilitation group

\begin{tabular}{lllllll} 
Training phase & Location & Week & $\begin{array}{l}\text { Weekly } \\
\text { sessions }\end{array}$ & $\begin{array}{l}\text { Aerobic duration (min) } \\
\text { of HRR) }\end{array}$ & $\begin{array}{l}\text { Intensity (percentage Mode of } \\
\text { of }\end{array}$ \\
\hline Initial, supervised by Rehabilitation specialist & Clinic & 2 & 3 & $15-30$ & 60 & Walk \\
supervised by Rehabilitation specialist & Clinic & 2 & 3 & $15-30$ & 70 & Walk \\
Supervised by remote ECG monitoring & Home & 8 & 5 & 40 & $60-70$ & Walk
\end{tabular}

HRR, heart rate reserve.

to monitor their own exercise training at the beginning of the trial. During the exercise, the monitor can evaluate whether patients have reached the intensity and time of the preset exercise prescription based on the heart rate. If the speed is not enough or the intensity exceeds the preset value, the system can remind the patient promptly to adjust the intensity, including speed and time course, to ensure that the patient to exercise based on the prescription. If arrhythmia occur during exercise, the system will alert the patient to stop or suspend exercise, or adjust the intensity of exercise, and upload it in real time. Specialists will give an analysis report. The system will give an early warning to both patients and their doctors if the arrhythmia is risky. Doctors can check data from patients outside hospital to understand their conditions and provide timely advice.

The heart rate reserve (HRR) method is used to guide exercise intensity. Peak heart rate is derived from a patient's most recent exercise test, and the resting heart rate is taken after a quiet seated rest for $5 \mathrm{~min}$. For the first six supervised training sessions, the training heart rate range is calculated as $60 \%$ of the HRR (resting heart rate +0.6 (peak heart rate-resting heart rate)). Then, training intensity is increased to $70 \%$ of the HRR for the rest of the supervised exercise training sessions. For the home-based exercise training phase, patients are asked to perform 40 min of aerobic exercise. In the REMS training arm, the training intensity should be maintained at $60 \%-70 \%$ of the HRR. The training intensity is not monitored during the entire phase of the conventional rehabilitation arm.

\section{Concomitant treatment}

Participants in both groups continue to receive standard treatment for HF. The medication should remain unchanged during the trial and the dosage should be adjusted in the event of adverse events. All procedures are determined by physicians following the clinical guidelines.

\section{Adherence}

During the training phase, participants are required to exercise in strict accordance with the prescription and use the designed cards to record each exercise process. Investigators make telephone reminders before each visit and then provide face-to-face reminders on each study visit to emphasise the importance of adherence. Compliance of ER in subjects is expressed in rate.

\section{Outcome assessments}

The primary outcome is an improvement in exercise capacity measured by peak oxygen uptake $\left(\mathrm{VO}_{2}\right.$ peak) (baseline vs $3 \mathrm{~m}$ ). The secondary outcomes are as follows: (1) difference in the metres walked in the $6 \mathrm{~min}$ walk test (6MWT); (2) improvement in heart function assessed by NYHA classifications; (3) improvement in echocardiographic parameters of systolic and diastolic function; (4) changes in biomarkers, including brain natriuretic peptide (BNP)/N-terminal-proBNP and Troponin-T/I; (5) changes in major adverse cardiovascular events including worsening HF event, HF hospitalisation, myocardial infarction, stroke, revascularisation and cardiovascular mortality; and (6) qualitative evaluation of patients' adherence to the rehabilitation programme. The investigators also use several validated psychometric instruments to measure health-related quality of life and depression. These measurements include HF Symptom Scale, Minnesota Living with Heart Failure Questionnaire, 36-Item Short Form Health Survey, Beck Depression Inventory-II and General Self-Efficacy Scale. Researchers involved in participants' assessments will be blinded to treatment allocation.

\section{Withdrawal}

According to Ethics Committee of Clinical Research legislations of China-Japan Friendship Hospital, we inform the patients about their rights as subjects in a scientific trial and about their rights to terminate. We do this to allow patients to fully consider participation thoroughly

Table 3 Exercise training programme in conventional rehabilitation group without monitoring

\begin{tabular}{|c|c|c|c|c|c|c|}
\hline Training phase & Location & Week & $\begin{array}{l}\text { Weekly } \\
\text { sessions }\end{array}$ & $\begin{array}{l}\text { Aerobic } \\
\text { duration (min) }\end{array}$ & $\begin{array}{l}\text { Intensity (percentage } \\
\text { of HRR) }\end{array}$ & $\begin{array}{l}\text { Mode of } \\
\text { exercise }\end{array}$ \\
\hline Initial, supervised by Rehabilitation specialist & Clinic & 4 & 3 & $15-30$ & Without monitoring & Walk \\
\hline
\end{tabular}

HRR, heart rate reserve. 
to reduce their likelihood of dropping out of the study. Patients may withdraw from the trial at their own request or at the request of their legal representative at any time.

\section{Data collection, management and analysis}

All patients' data are recorded by trained clinical researchers using a standardised case report form (CRF). Raw data should be recorded in timely and accurate manner. Copies of laboratory reports should also be kept. All CRFs are stored in locked file cabinets in areas with limited access. All laboratory specimens are identified by a coded number to maintain participant confidentiality. Data administrators from Cardiac Technology in Beijing, China are responsible for the data entry and management. The database was built using PHP language under Linux system. Two data managers independently perform dual input and proofreading to ensure data accuracy. The clinical research data management platform of China-Japan Friendship Hospital is responsible for data monitoring, which is independent of the study organisers. All individuals involved in data management and analysis are blinded to treatment allocation. Principal investigators have direct access to data sets. Data dispersed to project team members are blinded of any identifying participant information.

\section{Adverse events monitoring}

All adverse events that occurred during the 3-month study period will be reported in the final paper. A serious adverse event is defined as any untoward medical occurrence resulting in hospitalisation or which results in a life-threatening problem, death or disability. Adverse events are defined as any untoward occurrences in study participants, potentially related to implementation of the study protocol. All serious and unexpected adverse events will be reported to the Ethics Committee as required.

\section{Statistical analysis}

Continuous variables will be presented as the mean $\pm \mathrm{SD}$, median or IQR. Baseline characteristics of the cohort will be summarised using descriptive statistics. Whether there are imbalances will be analysed between groups. When normally distributed, independent samples will be compared for numerical variables between the two groups using Student's t-test. The categorical variables described as frequencies and percentages will be compared using $\chi^{2}$ test. Mann-Whitney $\mathrm{U}$ test will be used if data are not normally distributed.

Analysis of outcomes will be conducted based on the intention-to-treat principle. We will use three sets of analyses: full analysis set (FAS), per protocol set (PPS) and safety set. The FAS includes all randomised patients, and the PPS consists of all patients who complete the treatment protocol. The safety set consists of patients who receive at least one treatment with safety records after randomisation. Dropouts will be included in the analysis by modern imputation methods for missing data.

All statistical tests will be two-tailed. $\mathrm{p}<0.05$ is considered statistically significant. All statistical analyses will be completed by Beijing Zhengyuan Technology using SAS V.9.4 software.

\section{Patient and public involvement}

During the study design period, CHF patients and their relatives were invited to participate in surveys and discussions, which allowed us to know their strong desire in ER, especially home-based telerehabilitation. We also selected several patients to use the monitor, which helped us to identify problems in application. In addition, we invited medical specialists including cardiologists, rehabilitation therapists and statistical analysts to discuss the study design and revise the intervention method and outcome measures. The results of our study will be disseminated to patient and public involvement representatives and study participants who wish to be notified.

\section{DISCUSSION}

HERE-CHF evaluates the effect of cardiac telerehabilitation intervention that combines modern technology (sensor technology, internet and remote consultation) with evidence-based ER guidance strategies, including prevention of adverse events during exercising. The objective of this study is to investigate whether homebased cardiac ER using REMS is superior to conventional ER without monitoring in patients with CHF. We hypothesise that this intervention will improve physical activity levels and quality of life.

Home-based CR is a method to increase the ER participation rate in HF patients. Whether exercise prescription can be performed effectively and the safety in exercise are two main issues in home-based ER. Exercise intensity is the key of exercise prescription because the exercise must be appropriate. Heart rate is the most important factor to monitor the patient's exercise intensity. Our wearable ECG monitor can evaluate whether patients reach the intensity based on the heart rate and promptly alert the patients to ensure that exercise intensity is within the preset range. REMS will also detect risky arrhythmia and provide an early warning to both the patients and their doctors, which can encourage patients to overcome fear and adhere to exercise. The use of our REMS offers a prospect for the delivery and expansion of home-based cardiac ER programmes in HF patients beyond the supervised setting and will help to increase adherence, reduce risk factors and improve benefit-cost ratio, which may further enhance the effectiveness of ER.

CPET can be used to evaluate patient exercise capacity and exertional symptoms and can provide numerous physiological parameters. Multiple CPET-derived variables have been assessed for their association with mortality in systolic $\mathrm{HF}$ patients and peak $\mathrm{VO}_{2}$ is shown to be the strongest predictor of mortality. ${ }^{15}$ Therefore, we select it as the primary outcome in HERE-CHF. The 6MWT is chosen as a secondary outcome due to its useful prognostic information similar to peak $\mathrm{VO}_{2}{ }^{16}$ 6MWT is less expensive and much more convenient in comparison to the nontrivial 
costs of CPET with its distinctive value as a measure of routine activity. Quality of life and psychological state are important in the evaluation of home-based ER, which are also included in our study.

\section{CONCLUSION}

The HERE-CHF study will provide new insights into the effect of home-based cardiac ER guided by REMS. Our REMS will help to increase adherence and reduce risk factors for HF patients, which may further enhance the effectiveness of ER.

\section{Author affiliations}

1Department of Cardiology, China-Japan Friendship Hospital, Beijing, China ${ }^{2}$ Department of Integrative Cardiology, China-Japan Friendship Hospital, Beijing, China

${ }^{3}$ Department of Rehabilitation, China-Japan Friendship Hospital, Beijing, China ${ }^{4}$ Phase I Clinical Ward of Clinical Trial Research Center, China-Japan Friendship Hospital, Beijing, China

Acknowledgements The authors thank all the patients advisers and medical students for their assistance in the study.

Contributors XL, HJ, JL and PY conceived the original concept of the study, registered the trial and wrote the draft of the protocol manuscript. DF, XY, LZ, GC, YY, HL, LC, MS, CL, YL and YZ contributed to the design of the study. All the authors read and discussed the manuscript, and approved the final version.

Funding This work is financially supported by China Capital Health Development Research Special Fund (2018-2-4064). Inticare-MC-06 ECG monitors are provided by Tianjin Al-Life Medical Technology Co., Ltd, China. The funding source does not influence or comment on planned methods, protocol, data analysis or the draft report.

Competing interests None declared.

Patient consent for publication Not required.

Provenance and peer review Not commissioned; externally peer reviewed.

Open access This is an open access article distributed in accordance with the Creative Commons Attribution Non Commercial (CC BY-NC 4.0) license, which permits others to distribute, remix, adapt, build upon this work non-commercially, and license their derivative works on different terms, provided the original work is properly cited, appropriate credit is given, any changes made indicated, and the use is non-commercial. See: http://creativecommons.org/licenses/by-nc/4.0/.

\section{REFERENCES}

1. Chinese Society of Cardiology of Chinese Medical Association. Editorial Board of Chinese Journal of Cardiology. Guidelines for the diagnosis and management of chronic heart failure. Zhonghua Xin Xue Guan Bing Za Zhi 2007;35:1076-95.

2. Chen WW, Gao RL, Liu LS, et al. Hu SS Representative group of Chinese cardiovascular diseases report. Report on cardiovascular diseases in China (2017). Chinese Circulation Journal 2018;33:1-7.

3. Yancy CW, Jessup M, Bozkurt B, et al. 2013 ACCF/AHA guideline for the management of heart failure: a report of the American College of Cardiology Foundation/American Heart Association Task Force on Practice Guidelines. J Am Coll Cardiol 2013;62:e147-239.

4. Chinese Society of Cardiology of Chinese Medical Association. Editorial Board of Chinese Journal of Cardiology. Chinese guidelines for the diagnosis and treatment of heart failure 2014. Zhonghua Xin Xue Guan Bing Za Zhi 2014;42:98-122.

5. Chinese Rehabilitation Medical Association Professional Committee of Cardiovascular Diseases. Committee of Cardio-Cerebral-Vascular Diseases of GSC. Chinese expert consensus on cardiac rehabilitation for patients with chronic stable heart failure. Zhonghua Xin Xue Guan Bing Za Zhi 2014;42:714-20.

6. Ponikowski P, Voors AA, Anker SD, et al. 2016 ESC Guidelines for the diagnosis and treatment of acute and chronic heart failure: the Task Force for the diagnosis and treatment of acute and chronic heart failure of the European Society of Cardiology (ESC). Developed with the special contribution of the Heart Failure Association (HFA) of the ESC. Eur J Heart Fail 2016;18:891-975.

7. Keteyian SJ, Isaac D, Thadani U, et al. Safety of symptom-limited cardiopulmonary exercise testing in patients with chronic heart failure due to severe left ventricular systolic dysfunction. Am Heart $J$ 2009;158(4 Suppl):S72-7.

8. Abell B, Glasziou P, Hoffmann T. The contribution of individual exercise training components to clinical outcomes in randomised controlled trials of cardiac rehabilitation: a systematic review and meta-regression. Sports Med Open 2017;3:19.

9. Clark RA, Conway A, Poulsen V, et al. Alternative models of cardiac rehabilitation: a systematic review. Eur J Prev Cardiol 2015;22:35-74.

10. Lee $\mathrm{Y}$, Lee J, Seo H, et al. Effects of Home-based Exercise Training with Wireless Monitoring on the Left Ventricular Function of Acute Coronary Syndrome Patients. J Phys Ther Sci 2013;25:631-3.

11. Chung H, Ko H, Thap T, et al. Smartphone-Based Cardiac Rehabilitation Program: Feasibility Study. PLoS One 2016;11:e0161268.

12. Hwang R, Bruning J, Morris NR, et al. Home-based telerehabilitation is not inferior to a centre-based program in patients with chronic heart failure: a randomised trial. J Physiother 2017;63:101-7.

13. Di Lenarda A, Casolo G, Gulizia MM, et al. The future of telemedicine for the management of heart failure patients: a Consensus Document of the Italian Association of Hospital Cardiologists (A.N.M.C.O), the Italian Society of Cardiology (S.I.C.) and the Italian Society for Telemedicine and eHealth (Digital S.I.T.). Eur Heart J Suppl 2017;19(Suppl D):D113-29.

14. Chan AW, Tetzlaff JM, Altman DG, et al. SPIRIT 2013: new guidance for content of clinical trial protocols. Lancet 2013;381:91-2.

15. Keteyian SJ, Patel M, Kraus WE, et al. Variables Measured During Cardiopulmonary Exercise Testing as Predictors of Mortality in Chronic Systolic Heart Failure. J Am Coll Cardiol 2016;67:780-9.

16. Forman DE, Fleg JL, Kitzman DW, et al. 6-min walk test provides prognostic utility comparable to cardiopulmonary exercise testing in ambulatory outpatients with systolic heart failure. J Am Coll Cardiol 2012;60:2653-61. 
Correction: Effects of home-based cardiac exercise rehabilitation with remote electrocardiogram monitoring in patients with chronic heart failure: a study protocol for a randomised controlled trial

Li J, Yang P, Fu D, et al. Effects of home-based cardiac exercise rehabilitation with remote electrocardiogram monitoring in patients with chronic heart failure: a study protocol for a randomised controlled trial. BMJ Open 2019;9:e023923. doi: 10.1136/ bmjopen-2018-023923

This article was previously published with missing information.

Jiahui Li and Peng Yang are joint first authors.

Open access This is an open access article distributed in accordance with the Creative Commons Attribution Non Commercial (CC BY-NC 4.0) license, which permits others to distribute, remix, adapt, build upon this work non-commercially, and license their derivative works on different terms, provided the original work is properly cited, appropriate credit is given, any changes made indicated, and the use is non-commercial. See: http://creativecommons.org/licenses/by-nc/4.0/.

(c) Author(s) (or their employer(s)) 2020. Re-use permitted under CC BY-NC. No commercial re-use. See rights and permissions. Published by BMJ.

BMJ Open 2020;10:e023923corr1. doi:10.1136/bmjopen-2018-023923corr1

A) Check for updates 